\title{
Security of Photo Sharing on Online Social Networks
}

\author{
Harshali Chandel ${ }^{1}$, Dr. A. M. Bagade ${ }^{2}$ \\ ME Student, Dept. of Information Technology, PICT, Pune, India ${ }^{1}$ \\ Assistant Professor, Dept. of Information Technology, PICT, Pune, India ${ }^{2}$
}

\begin{abstract}
Photo sharing is an attractive feature in Online Social Networks (OSNs) but it may leak users privacy if they are allowed to post, comment, and tag a photo freely. This issue of sharing the photos of individual or himself/herself is addressed by the proposed scheme. The proposed scheme is used to prevent possible privacy leakage of a photo. For this purpose, an efficient facial recognition (FR) system is required that can recognize everyone in the photo. However, to train the FR system, more demanding privacy setting may limit the number of the photos that are publicly available. To solve this problem, the proposed scheme attempts to utilize users private photos by designing a personalized FR system and also provide security while posting the photo. A distributed consensus based method is also developed to reduce the computational complexity and protect the private training set. The efficiency of proposed scheme is calculated by using recognition ratio. The proposed mechanism is implemented as a proof of concept on Android application in OSN's (Online Social Networks) on Facebook platform.
\end{abstract}

Keywords: social networks, photo privacy.

\section{INTRODUCTION}

With the huge popularity of sharing and the vast usage of social networking sites users unknowingly reveal certain kinds of personal information. Social-networking users may or may not have the idea of getting their personal information will be leaked or could profit the malicious attackers and may perpetrate significant privacy breaches. The first decade of 21st century has seen the extreme popularization of Internet and the growth of web services which facilitate participatory information sharing and collaboration. Social Networking Sites (SNSs) have become a boundless communication media to keep in touch beyond boundaries. SNSs are a part of human culture than just a web application. Use of SNSs has out spaced in almost every fields as news agencies, big and small companies, governments, and famous personalities etc. to interact with each other. With the adoration of sharing, Facebook has stood out as the most renown SNSs in the world where people hangout for hours. With the extravagancy of technology and services sharing of news, photos, personal taste and information with friends and family has lead to an ease. But along with this user privacy should also be taken into consideration [1].

An issue related to privacy with Facebook users has been constantly appearing on international press either because of the companies privacy policy or because of users unawareness of content sharing consequences. As a research says the simple disclosure of date and place of birth of a profile in Facebook can be used to predict the Social Security Number (SSN) of a citizen in the U.S. Many a times just by simply publishing their friends list, users might be revealing a large amount of information. For example, through the use of prediction algorithms it is possible to infer private information that was previously undisclosed. Sometimes sensitive information even comes embedded in the photo as metadata and may identify people on the photo by accompanying more information that could be exploited, like captions, comments and photo tags; marked regions. Even if the individuals in a photo are not explicitly identified by photo tags, the combination of publicly available information and face recognition software can be used to infer someones identity. These kinds of problems are defined as collateral damage: users unintentionally put their own privacy or their friends privacy at risk when performing events on SNSs such as Facebook [2].

With the ease and need of fulfilling our social needs social interactions, information sharing, appreciation and respect Social Networking sites have became the integral part of daily life. With this ease and nature of social media people put more content, including photos, over OSNs without too much thought on the content. Once a photo is posted online it becomes a permanent record which may further be used for malicious purposes. For example, a posted photo in a party may reveal a connection of a celebrity to a mafia world. As OSN users may be careless in posting content while the effect is so far-reaching, privacy protection over OSNs becomes an important issue.

Another features of the Social networking Sites like photo tagging etc may create more complications when user privacy come in concerns. So far there is no restriction with sharing of co-photos, on the converse, social network service providers like Facebook are encouraging users to post co-photos and tag their friends in order to get more people involved. This project proposes a system based on novel consensus, approach to achieve efficiency and privacy 
at the same time. The main focus is to let each user only deal with his/her private photo set as the local train data which can be used by the users to learn out the local training result. Once the local training results are achieved then it can be exchanged among various users to form a global knowledge. In the next round, each user learns over his/hers local data again and takes the global knowledge as a reference. Finally the information is spread over users and consensus can be reached[10].

\section{RELATED WORK}

N. Mavridis, w. Kazmi, and p. Toulis[1] Study the statistics of photo Sharing on social networks and propose a three realms Model: "a social realm, in which identities are entities, And friendship a relation; second, a visual sensory realm, Of which faces are entities, and co-occurrence in images A relation; and third, a physical realm, in which bodies Belong, with physical proximity being a relation." They show that any two realms are highly correlated. Given information in one realm, we can give a good Estimation of the relationship of the other realm.

Z. Stone, t. Zickler, and t. Darrell[2] Propose to use The contextual information in the social realm and cophoto Relationship to do automatic fr. They define a Pairwise conditional random field (crf) model to find The optimal joint labeling by maximizing the conditional Density. Specifically, they use the existing labeled photos As the training samples and combine the photo cooccurrence Statistics and baseline fr score to improve The accuracy of face annotation.

K. Choi, h. Byun, and k.-a. Toh [3] Discuss the difference between the traditional FR system and the FR system that is designed specifically for OSNs. They Point out that a customized FR system for each user is Expected to be much more accurate in his/her own photo Collections.

J. Y. Choi, w. De neve, k. Plataniotis, and y.-m[4] propose a novel collaborative face recognition (FR) framework, improving the accuracy of face annotation by effectively making use of multiple FR engines available in an OSN. Their collaborative FR framework consists of two major parts: selection of FR engines and merging (or fusion) of multiple FR results. The selection of FR engines aims at determining a set of personalized FR engines that are suitable for recognizing query face images belonging to a particular member of the OSN. For this purpose, they exploit both social network context in an OSN and social context in personal photo collections. In addition, they devise two effective solutions for merging FR results, adopting traditional techniques for combining multiple classifier results.

D. Rosenblum[5] The privacy leakage caused by The poor access control of shared data in web 2.0 is Well studied. C. Squicciarini, m. Shehab, and f. Paci [6] Propose a Game-theoretic scheme in which the privacy policies are collaboratively enforced over the shared data. Each user is able to define his/her privacy policy and exposure Policy. Only when a photo is processed with owner's Privacy policy and co-owner's exposure policy could it be posted.

K. Thomas, C. Grier and D. M. Nicol [7] examine how the lack of joint privacy controls over content can inadvertently reveal sensitive information about a user including preferences, relationships, conversations, and photos. They analyze Facebook to identify scenarios where conflicting privacy settings between friends will reveal information that at least one user intended remain private. they show how Facebook's privacy model can be adapted to enforce multi-party privacy and present a proof of concept application built into Facebook that automatically ensures mutually acceptable privacy restrictions are enforced on group content.

A. Besmer and H. Richter Lipford [8] examine privacy concerns and mechanisms of tagged images. Using a focus group they explored the needs and concerns of users for tagged photo privacy. They also designed a privacy enhancing mechanism and validated it using a mixed methods approach. Their results identify the social tensions that tagging generates, and the needs of privacy tools to address the social implications of photo privacy management.

\section{RESEARCH ELABORATION}

Proposed system is used to maintain privacy of user on online social networking sites. User used $\log$ in/out button for $\log$ in/out with Facebook. After logging in, a greeting message and the profile picture will be shown. The proposed system works in three modes: a setup mode, a sleeping mode and a working mode. For running in the setup mode, the program is established the decision tree. For this purpose, User should specified his private training set and neighborhood set. Private training set could be specified by the user with the button "Private training set".

When it is pressed, photos in the smart phone galleries could be selected and added to that set and for the the neighborhood set, user needs to manually specify the set of "close friends" among their Facebook friends with the button "Pick friends" as their neighborhood. In this way the setup mode could be activated by pressing the button "Start". After the classifiers are obtained, decision tree is constructed and the program switches from the setup mode to the sleeping mode. According to the proposed scheme, the friend list should be intersection of owner's privacy policy and co-owners' exposure policies. By clicking on "Post Photo", the co-owners of that photo will be identified and then notification will be send to them and with their permission the photo will be post and user could also specify the policy on that photo[1]. 


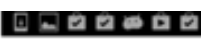

If Log in with Facebook

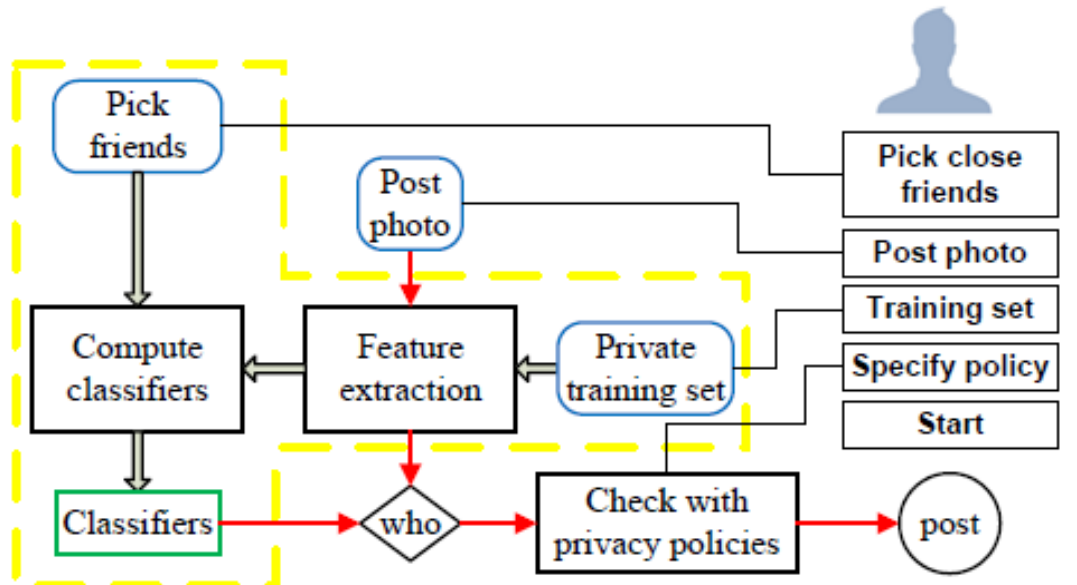

Fig 1: Architecture model for the proposed system[1].

\section{Modules:}

\section{Friend Request}

A $\log$ in/out button could be used for log in/out with the social website. After logging in, a greeting message and the profile picture will be shown. This prototype works in three modes: a setup mode, a sleeping mode and a working mode.

\section{Picking Close Friends}

A user needs to manually specify the set of "close friends" from their friend list on social website and form the neighborhood by clicking the button "Pick friends".

\section{Sharing Photo}

User can share a photo only to friends on list. According to the proposed scheme, this friend list should be intersection of owner's privacy policy and co-owners' exposure policies.

\section{Face Detection and Face Recognition}

Face detection is based upon the training of a classifier using number of positive images that represent the object to be recognized and even large number of negative images that represent objects or feature not to be detected. The photo $\mathrm{x}$ is provided in which I faces are detected. This technique can be adapted to accurately detect facial features. The area of the image is regionalized containing the highest probability of the feature so as to analyze the facial feature.

\section{Check Policy Status}

The privacy policy status is set for individual users. The policy should satisfy both the privacy policy and the exposure policy of the individuals.

\section{Post or Block}

If the policy is satisfied then the notification is sent to the co-owner. The photo is posted once the owner gives permission to upload it else it is not uploaded.

\section{DISCUSSION}

Photo sharing is one of the most popular features in online social networks such as Facebook. Photo sharing is the process of publishing or transfer of a user's digital photos online.To control the privacy leakage, this project proposed the FR system to identify the individuals in a co-photo. After identifying the individual, with their permission the photo will be post. The proposed system is featured with low computation cost and confidentiality of the training set. Various websites offer services such as uploading, hosting, and managing for photo-sharing (publicly or privately). These functions provided by websites and applications facilitate the upload and display of images. The term may even be useful for online photo galleries that are positioned up and managed by individual users, including photo blogs. 


\section{CONCLUSION}

Photo sharing is the process of publishing or transfer of a user's digital photos online.To control the privacy leakage, this paper proposes the FR system to identify the individuals in a co-photo. After identifying the individuals, with their permission the photo will be posted. The proposed system is featured with low computation cost and confidentiality of the training set. It proposes a privacy-preserving FR system to identify individuals in a co-photo which is very useful in protecting users' privacy in photo/image sharing over online social networks. This proposed system can be created as an Android application in which photo could only be posted with the permission of all co-owners. Latency introduced in this process will greatly impact user experience of OSNs.

\section{REFERENCES}

[1] N. Mavridis, W. Kazmi, and P. Toulis. Friends with faces: How social networks can enhance face recognition and vice versa. In Computational Social Network Analysis, Computer Communications and Networks, pages 453-482. Springer London, 2010.

[2] Z. Stone, T. Zickler, and T. Darrell. Toward large-scale face recognition using social network context. Proceedings of the IEEE, 98(8):14081415,2010 .

[3] K. Choi, H. Byun, and K.-A. Toh. A collaborative face recognition framework on a social network platform. In Automatic Face Gesture Recognition, 2008. FG '08. 8th IEEE International Conference on, pages 1-6, 2008.

[4] J. Y. Choi, W. De Neve, K. Plataniotis, and Y.-M. Ro. Collaborative face recognition for improved face annotation in personal photo collections shared on online social networks. Multimedia, IEEE Transactions on, 13(1):14-28, 2011.

[5] D. Rosenblum. What anyone can know: The privacy risks of social networking sites. Security Privacy, IEEE, 5(3):40-49, 2007.

[6] C. Squicciarini, M. Shehab, and F. Paci. Collective privacy management in social networks. In Proceedings of the 18th International Conference on World Wide Web, WWW'09, pages 521-530, New York, NY, USA, 2009.

[7] K. Thomas, C. Grier, and D. M. Nicol. unfriendly: Multi-party privacy risks in social networks. In M. J. Atallah and N. J. Hopper, editors, Privacy Enhancing Technologies, volume 6205 of Lecture Notes in Computer Science, pages 236-252. Springer, 2010.

[8] Besmer and H. Richter Lipford. Moving beyond untagging: photo privacy in a tagged world. In Proceedings of the SIGCHI Conference on Human Factors in Computing Systems, CHI '10, pages 1563-1572, New York, NY, USA, 2010.

[9] P. A. Forero, A. Cano, and G. B. Giannakis. Consensus-based distributed support vector machines. J. Mach. Learn. Res., 99:1663-1707, August 2010.

[10] Z. Stone, T. Zickler, and T. Darrell. Autotagging facebook: Social network context improves photo annotation. In Computer Vision and Pattern Recognition Workshops, 2008. CVPRW'08. IEEE Computer Society Conference on, pages 1-8. IEEE, 2008.

\section{BIOGRAPHIES}

Harshali Ashok Chandel is a student of Master of Engineering (M.E), Information Technology Branch in Pune Institute of Computer Technology (PICT), Pune.

Dr. Anand Bagade is a Professor in the Information Technology Department, Pune Institute of Computer Technology (PICT), Pune. He has received Ph.D. in computer Science and Engineering. 